\title{
Usefulness of Nerve Blocks and Radiofrequency Treatment in Nonagenarians with Chronic Radicular and Low Back Pain : A Preliminary Study
}

\author{
Sang-In Yoon, Tae-Shin Kim, Jang-Bo Lee, Jung Yul Park ${ }^{凶}$ \\ Department of Neurosurgery, Korea University Medical Center, College of Medicine, Korea University, Seoul, Korea
}

\begin{abstract}
Objective: The purpose of this study is to evaluate the usefulness of minimal invasive interventional treatments with series of nerve blocks and radiofrequency (RF) treatment on dorsal root ganglion(s) and medial branches in patients aged 90 years and older who suffered from chronic radicular and back pain. Methods: A total of 10 consecutive patients who were treated conservatively within recent one year period were included in this study. All patients were over 90 years of age with chronic low back and radicular pain, without major neurological deficits, for more than six months that were refractory to medication and rehabilitation treatment. All patients had combination of various pathologies including multiple spinal stenosis, herniated discs, and facet arthropathies. Treatments comprised of series of nerve block (medial branch blocks) with or without radiofrequency lesionings (RF neurotomy on medial branch and pulsed RF dorsal root ganglionotomy). Those who had recurrent pain after two nerve blocks which showed more than $50 \%$ pain relief for at least 2 weeks following two nerve blocks also received RF treatment. Follow up period was at least six months after treatments. Results: There were 4 patients who received nerve blocks only and 6 patients who also received RF treatment. Average baseline pain severity was 6.7 (range 4-9). Six months after the procedures in patients who received both nerve blocks and RF treatment, 1 patient showed excellent (>75\% reduction of baseline pain), 3 patients reported good (>50\%) results, and remaining 2 patients showed moderate response $(>30 \%)$. There were no patients with poor $(<30 \%)$ or no response and no complications from the procedures except moderate pain following the RF procedures in 4 patients that persisted for average of 4.7 days. All patients are functionally stable at last follow up period. Conclusion: Series of nerve blocks with or without RF treatment seem to provide substantial pain relief with satisfaction even in very old patients with chronic low back pain and radicular pain from various mixed pathologies. Although longer term follow up with more patients is warranted in future study, it can thus be considered as safe and effective treatment option before selecting surgery with a reasonable tradeoff in terms of pain reduction and functional disability improvement.
\end{abstract}

Key Words: Nonagenarians; Nerve block; Radiofrequency; Lesioning; Mechanical back pain.

\Corresponding author: Jung Yul Park, Department of Neurosurgery, Korea University Medical Center, College of Medicine, Korea University, 73 Inchon-ro, Seongbuk-gu, Seoul 02841, Korea. Tel: 82-2-920-5729, Fax: 82-2-929-0629, E-mail: jypark98@korea.ac.kr

\section{INTRODUCTION}

In 2012, life expectancy of Korea was 84.6 in female, and 77.9 in male and these values were all higher than mean life expectancy of OECD countries. As the average life span expands, the concern for improving quality of life is required. Chronic pain is one of the most disabling and common symptom that causes reduction in quality of life ${ }^{5}$. Pain also reduces mobility of individuals and causes loss of interest in living with depressed mood ${ }^{6}$. Furthermore, it causes great burden in nation's economy due to its cost for medical treatment ${ }^{7)}$. Persistent pain costs approximately 600 billion dollars annually in USA ${ }^{4)}$, so controlling chronic pain with methods that discharge low fee should be one of national tasks to resolve. This is especially true when the cost for medical treatment for elderly population is steeply increasing in recent years with need for long term care for these people as their life expectancy is continuously increasing.

One of common pain symptom that can be seen in elderly patients is chronic back pain. Treatment options for back pain patients include medication therapy, pain management therapy, 
and surgical therapy. Old age patients usually have more than one comorbidities so surgical treatment may be too heavy to burden $^{2,12,14)}$. Also, the correlation between radiological imaging findings and clinical symptoms are often mismatch. Many patients with significant spinal stenosis and other structural abnormalities tolerate well despite the severity of their pathologic changes. Thus, series of less invasive treatment options, such as nerve blocks, should be first considered first before surgery based on radiological findings. The nerve blocks have long been treatment of option for those who do not have substantial pain relief or development of recurrent pain after series of short term beneficial effect. They mostly consist of medial branch block to relieve pain from facet joints, paravertebral muscles, and interspinous ligaments. Also, transforaminal epidural block or dorsal root ganglion block is to relieve radicular pain as well as pain originated from disc.

Radiofrequency (RF) neurotomy on medial branches with or without and gangliotomy has been considered to be alternative methods for treatment of chronic low back pain in patients with refractory to conservative treatment and recurrent pain following series of nerve blocks. RF treatment is minimally invasive treatment that is used for reducing noxious transmission in the nervous system without causing permanent destructive changes on neural element, especially the dorsal root ganglion. Current $\mathrm{RF}$ technique for treating low back pain and radicular pain consists of a conventional, continuous RF neurotomy on medial branch (with heat of $80 \mathrm{C}$ ) and pulsed RF ganglionotomy on DRG (with heat of $42 \mathrm{C}$ ) based on several studies of RF treatment to prove its effectiveness ${ }^{3,8,11,13)}$. However, its long term effectiveness and feasibility for very old, the nonagenarians (more than 90 years of age), patients have not been reported. The purpose of this study is to evaluate the effect and usefulness of nerve blocks and RF treatment applied to the medial branch and dorsal root ganglion in nonagenarians with chronic low back pain and radicular pain.

\section{MATERIALS AND METHODS}

Our study was comprised of 10 patients who underwent nonsurgical management for chronic back and radicular pain. Eligible patients were at least 90 years of age who received series of nerve blocks with or without RF treatment; had experienced more than 6 months of low back pain and who were followed up for more than six months after first treatment. All of these had at least one medical comorbidity such as diabetes, hypertension, COPD, and renal dysfunction and each patient has average 2.8 comorbidities.

All patients had combination of various pathologies including multiple spinal stenosis, herniated discs, and facet arthropathies with varying degrees of severity. Nine of these patients had multiple lesions and 8 had more than two levels of involvement. These patients had received conservative treatments for at least 3 months from other institution without substantial pain relief before referred to our hospital.

Four patients (two males and two females) received series of nerve blocks and remained as much improved state (2 patients) without RF treatment or did not want RF treatment as they were satisfied with the status or fear of procedure (2 patients). These patients are now visiting outpatient regularly with medications and rehabilitation program. Remaining six patients received RF treatment that consisted of RF medial branch neurotomy and pulsed RF dorsal root ganglionotomy as described elsewhere ${ }^{1,9,10)}$ Briefly, with the guide of C-arm radiographic image intensifier, the level and target of the procedure were verified with patient on prone. For the medial branch block, 23G spinal needle was inserted maintaining the angle about 10-15 degrees between the needle and horizontal plane, and advanced to the point encountered with superior articular process and transverse process (Burton's point). The tip was then redirected in a slightly more cephalad and lateral direction until contact with bone was lost. Because of innervation of the each facet joint is derived from at least two spinal levels, one above and one below, these were performed at three levels for each joint. After confirming the targets, $2 \mathrm{cc}$ of mixed solution consisted of steroid, lidocaine and hyaluronidase, was injected to each target. For DRG block, a 23-gauge spinal needle was introduced to the target point, which was a so called safe triangle, i.e., above the nerve root and below the pedicle until bony contact was encountered. After verifying correct needle positioning under AP fluoroscopic control, contrast solution was injected to confirm that the material was passing the selected nerve root and was not administered intravascularly. The finally verified image was recorded. Subsequently, $3 \mathrm{~mL}$ of mixed solution were injected into each targeted point. Following the procedure, the needle was withdrawn, the site was sealed with a sterile dressing, and the patients were monitored for 30 minutes for any untoward side effects. For RF neurotomy on medial branch of posterior primary ramus, procedure was same except insertion of RF needle (SMK-C10, 22-gauge, $100 \mathrm{~mm}$ long, $5 \mathrm{~mm}$ active tip) followed by readjustment according to resistance of tissue and the thresholds of sensory and motor responses from electrical stimulation $(50 \mathrm{~Hz}$ and $2 \mathrm{~Hz}$, respectively). Once the position of the electrode was satisfactory, RF neurotomy was performed at $80^{\circ} \mathrm{C}$ for 60 seconds using RF lesion generator (NeuroThermTM JK25T, RDG Medical Inc., Surrey, UK). For pulse RF dorsal root ganglionotomy, procedures were similar except electrode tip was placed near $(<2 \mathrm{~mm})$ DRG by transforaminal approach through safe triangle (Fig. 1). After confirmation of correct placement by contrast dye and physiological monitorings, final lesioning is accomplished with temperature set to 42 $\mathrm{C}$ for 4 minutes.

For those who were treated with RF, there were 2 males (33.3\%) and 4 females (66.6\%). The age ranged from $90-97$ years (mean 91.3 years). The medical records of patients were carefully reviewed retrospectively. The assessments included the clinical symptoms, and Visual analog scale (VAS) was used to measure 


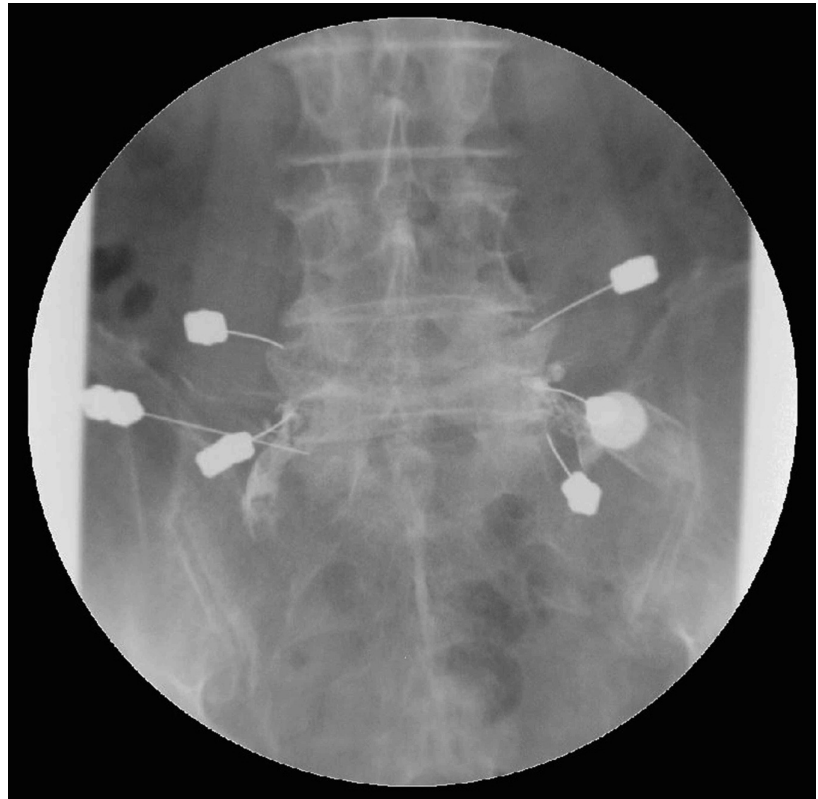

Fig. 1. An intraoperative radiograph showing RF lesining on the medial branches of the posterior rami and dorsal root ganglia.

subjective pain levels before and after RF procedure.

We used the VAS for evaluating the pain intensity before and after surgery. Pain-free result was considered as a complete resolution of pain, excellent result was considered to be at least $75 \%$ relief in pain, good result was more than $50 \%$ relief in pain, moderate effect more than $30 \%$ relief, and poor as less than $30 \%$ relief. We considered pain-free, good, excellent and moderate results as clinically significant reduction in pain. The patients were examined three times during the first year : one, three, six months after the procedure.

\section{Statistical analysis}

Averages were calculated for the different variables. Differences were compared using the one-way analysis of variance test. $p$-value $<0.05$ were considered to be significant.

\section{RESULTS}

As described above, four patients (two males and two females) received series of nerve blocks and remained as much improved state (2 patients, more than 50\%) without RF treatment or did not want RF treatment as they were satisfied with the status or fear of procedure ( 2 patients). These patients are now visiting outpatient regularly with medications and rehabilitation program. Remaining six patients received RF treatment. All of these patient had reported varying degrees of improvement at the 1 month, 3 months and 6 months follow-up. However, at the first evaluation, no patient reported more than 50\% reduction of pain, 4 patients were classified as moderate, and 2 patients showed mild effect. At the second evaluation, 1 patient reported to feel better than before, 3 remained moderate class, and 2 obtained mild effect. At the third follow up evaluation, 1
Table 1. Results after RF treatment

\begin{tabular}{lcccc}
\hline \multirow{2}{*}{$\begin{array}{c}\text { Post RF } \\
\text { follow up }\end{array}$} & \multicolumn{4}{c}{ Pain relief level } \\
\cline { 2 - 5 } 1 month & Significant-effect & Moderate & Minimal-effect & Total \\
\hline 3 months & 1 & 4 & 2 & 6 \\
6 months & 1 & 3 & 2 & 6 \\
\hline
\end{tabular}

Table 2. Mean VAS before and after RF treatment

\begin{tabular}{cccc}
\hline Before RF & \multicolumn{3}{c}{ After RF procedures } \\
\cline { 2 - 4 } procedures & 1 month & 3 months & 6 months \\
\hline 6.7 & 3.3 & 3.3 & 2.7 \\
\hline
\end{tabular}

patient remained to have significant effect, 2 patients remained to show moderate reduction, and 3 patients had mild VAS score improvement was observed in all patients (Table 1). Average reduction in VAS score was 3.3 (baseline VAS 6.7) after 1 months, 3.3 after 3 months, and 2.7 after 6 months. There were no complications from the procedures except moderate pain following the RF procedures in 4 patients that persisted for average of 4.7 days. Although these patients are taking analgesics with mild to moderate potency once or twice a day (none under opiate analgesics), all of these patients are satisfied with the treatments and are visiting OPD clinic every 1-3 months depending on their symptoms (Table 2).

\section{DISCUSSION}

Increasing age has been associated with an increase in musculoskeletal symptoms such as low back pain with or without radicular leg pain. LBP. The pathophysiological mechanisms are complex especially in elderly patients and these are usually mixed with varying degrees of severity. On the lumbar level, the posterior compartment of the spine is innervated by the posterior primary ramus of segmental nerve. The medial branch of the posterior primary rami innervates the posterior joints in such a way that each joint is served by at least three segmental levels. The posterior zygapophyseal joints (facet joint) of spine have long been considered responsible for some cases of mechanical neck and back pain. 1 The estimated prevalence of lumbar facet joint pain among patients with chronic LBP rages from 15 to $40 \%^{5,7)}$.

Currently, the most commonly and approved methods to relieve such pain are injection of local anesthetic and steroids in and around the joints and the RF ablation of their nerve supply when refractory to conservative treatment. Dorsal root ganglion is known to be important in transmission and modulation painful impulse to dorsal horn of spinal cord as well as production of neurotransmitters or inflammatory mediators related to pain. In spinal disorders, it is usually involved in causing radicular pain or radiculopathy. Thus, it has become a major target interest when treating patients with such symptoms. In case of the specific lesion that matched patient's symptoms, and if these symptoms persist despite intensive conservative treatment, sur- 
gery could be a good choice for improving LBP. But invasive surgery may increase morbidity and mortality with increasing age, especially older than 65 years due to their combined medical diseases and poor physical conditions. Several studies have been done to assess the risk of morbidity and mortality occurring in elderly patients undergoing spinal surgery ${ }^{3,5}$. Results of these studies indicated increased morbidity and mortality with increasing age, and reported that mortality rates increase significantly as the number of comorbidities increased. Therefore, the greater cautions should be given when surgery is considered in elderly patients with respect to conditions for general anesthesia, general health status, comorbidities, benefit-risk rates, and cost-effectiveness.

Since Uematsu et al. first reported the percutaneous RF lesioning of the DRG in 1974, and Shealy first described percutaneous RF facet denervation in $1975^{10)}$, there have been not only the great advancement, in recent years, of knowledge on pain but also the refinement of hard-wares and techniques related to $\mathrm{RF}$ treatment ${ }^{3,5,8-10)}$. These minimally invasive treatments for managing LBP were adopted in this study for their convenience, safety, and effectiveness. This is very important for elderly patients because of relatively high success rates of improvement without surgical interventions and unwanted side effects. Other advantages are its repeatability, cost-effectiveness, and feasibili$\operatorname{ty}^{11)}$. However, its long term effectiveness and feasibility for very old, the nonagenarians (more than 90 years of age), patients have not been reported. Thus, the purpose of this study was to evaluate the effect and usefulness of nerve blocks and RF treatment targeted to the medial branch and dorsal root ganglion in nonagenarians with chronic low back pain and radicular pain.

As elderly patients usually accompany with medical diseases, their physical and immunity conditions are usually poor. In our study, all patients had at least one medical comorbidity such as diabetes, hypertension, COPD, and renal dysfunction and each patient has average 2.8 comorbidities. This is a heavy burden for elderly patients, thus validation of therapeutic effects of nerve block and RF treatment may be quite meaningful in these patients with such clinical settings. In this respect, our study results indicate that minimally invasive treatment such as nerve block and RF treatment could be alternative choices for elderly patients in managing chronic LBP and radicular pain for at least six months after the treatments. Another bright side of these treatments is that these treatments can be repeated when patients experience the recurrence of symptoms. Nerve blocks can be repeated 2-3 times a year and RF treatment every six months without any serious side effects when precisely performed in selected patients. In our study, although short in follow up period with small number of patients, there were no complications except mild low back pain for average 4.7 days following RF procedures. In this study, we didn't demonstrate the effectiveness of repeated RF procedure, but one patient who had significant reduction mentioned above have received RF procedure twice at interval of 6 months with similar degrees of pain relief.

The issue of prognostic blocks prior to the definitive procedure was concerned in previous articles ${ }^{9,15}$. We performed at least two times of prognostic/therapeutic blocks before every RF procedures. Prior studies mention prognostic blocks can produce false-positive and false negative results (but in our case, prognostic/therapeutic blocks helped to classify patients who didn't require RF procedure). Moreover, four or our patients received series of nerve blocks and remained as good improvement state without RF treatment or did not want RF treatment as they were satisfied with the status.

This study has some limitations. The number of patients was small and follow up period was only six months. Also, there was no control group for the comparison. However, this is the first report of usefulness of nerve blocks and RF treatment in patients over 90 years of age with chronic refractory low back and radicular pain and all patients who have received procedure had substantial reduction in pain with satisfaction for at least six month of follow up.

\section{CONCLUSION}

Series of nerve blocks with or without RF treatment seem to provide substantial pain relief with satisfaction even in very old patients with chronic low back pain and radicular pain from various mixed pathologies. Although longer term follow up with more patients is warranted in future study, it can thus be considered as safe and effective treatment option before selecting surgery with a reasonable tradeoff in terms of pain reduction and functional disability improvement.

\section{REFERENCES}

1. Bogduk N, Long DM : The anatomy of the so-called "articular nerves" and their relationship to facet denervation in the treatment of low-back pain. Journal of Neurosurgery 51 : 172-177, 1979

2. Choma TJ, Schuster JM, Norvell DC, Dettori JR, Chutkan NB : Fusion versus nonoperative management for chronic low back pain : do comorbid diseases or general health factors affect outcome? Spine 36 : S87-S95, 2011

3. Dreyfuss P, Halbrook B, Pauza K, Joshi A, McLarty J, Bogduk N : Efficacy and validity of radiofrequency neurotomy for chronic lumbar zygapophysial joint pain. Spine $25: 1270-1277,2000$

4. Gaskin DJ, Richard P : The economic costs of pain in the United States. J Pain 13 : 715-724, 2012

5. Gore M, Sadosky A, Stacey BR, Tai KS, Leslie D : The burden of chronic low back pain : clinical comorbidities, treatment patterns, and health care costs in usual care settings. Spine 37 : E668-677, 2012

6. Kaptan H, Yalcin ES, Kasimcan O : Correlation of low back pain caused by lumbar spinal stenosis and depression in women : a clinical study. Archives of Orthopaedic and Trauma Surgery $132: 963-967,2012$

7. Manchikanti L, Boswell MV, Singh V, Benyamin RM, Fellows B, Abdi S, et al. : Comprehensive evidence-based guidelines for interventional techniques in the management of chronic spinal pain. Pain Physician 12: 699-802, 2009

8. Masala S, Nano G, Mammucari M, Marcia S, Simonetti G : Medial branch neurotomy in low back pain. Neuroradiology 54 : 737-744, 2012.

9. North RB, Han M, Zahurak M, Kidd DH : Radiofrequency lumbar facet 
denervation : analysis of prognostic factors. Pain 57 : 77-83, 1994

10. Shealy CN : Percutaneous radiofrequency denervation of spinal facets. Treatment for chronic back pain and sciatica. Journal of Neurosurgery 43 : 448-451, 1975

11. Son JH, Kim SD, Kim SH, Lim DJ, Park JY : The efficacy of repeated radiofrequency medial branch neurotomy for lumbar facet syndrome. Journal of Korean Neurosurgical Society 48 : 240-243, 2010

12. Strong WE, Blanchard J, Ramamurthy S, Hoffman J : Does the sympathetic block outlast sensory block : a thermographic evaluation. Pain 46 : 173-176, 1991
13. van Wijk RM, Geurts JW, Wynne HJ : Long-lasting analgesic effect of radiofrequency treatment of the lumbosacral dorsal root ganglion. Journal of Neurosurgery $94: 227-231,2001$

14. Wetzel FT, LaRocca SH, Adinolfi M : The treatment of chronic extremity pain in failed lumbar surgery. The role of lumbar sympathectomy. Spine $17: 1462-1468,1992$

15. Yilmaz C, Kabatas S, Cansever T, Gulsen S, Coven I, Caner H, et al. : Radiofrequency facet joint neurotomy in treatment of facet syndrome. Journal of Spinal Disorders \& Techniques 23 : 480-485, 2010 